\title{
De 2006 à 2014: a trajetória dos brasileiros no ICPAPH
}

\section{From 2006 to 2014: the trajectory of Brazilians in the ICPAPH}

\author{
Claudia 0 Alberico \\ Felipe V Cureau ${ }^{2}$ \\ Paola M Duarte ${ }^{3}$ \\ Priscila B Gonçalves \\ Grégore I Mielke ${ }^{4}$
}

\section{RESUMO}

A participação em eventos científicos é uma oportunidade para muitos pesquisadores trocarem experiências, ideias e criarem parcerias. Com o objetivo de ampliar a discussão sobre questões relacionadas à atividade física e saúde pública, foi criado o International Congress on Physical Activity and Public Health (ICPAPH). A pesquisa epidemiológica em atividade física e saúde cresce no Brasil, assim como a participação de brasileiros em sociedades e eventos internacionais. O objetivo deste estudo é descrever a participação de brasileiros em todas as edições do ICPAPH realizadas até 2014, ano em que o evento foi realizado no Brasil. Através da análise dos anais dos congressos, assim como informações obtidas através dos presidentes de cada edição e relatos de brasileiros, observou-se que a participação do Brasil ocorre desde a primeira edição em 2006. Cerca de 2\% da participação geral de palestrantes tem sido de brasileiros, assim como $5 \%$ das apresentações de trabalhos, com aumento dessas participações na edição realizada no Rio de Janeiro (14\% e 40\%, respectivamente), o que se esperava devido à grande participação de congressistas locais em todas as edições do evento $(2006=68 \%, 2008=14 \%, 2010=48 \%, 2012=60 \%$ e $2014=32 \%)$. Sendo assim, apesar do crescimento na participação de brasileiros neste evento internacional, estratégias de incentivo à participação de pesquisadores brasileiros em outros eventos de discussão internacional e de fomento à pesquisa na área de atividade física e saúde pública devem ser elaboradas.

\section{PALAVRAS-CHAVE}

Pesquisa; Academias e institutos; Eventos científicos e de divulgação.

\begin{abstract}
The participation in scientific events is an opportunity for many researchers to exchange experience, ideas and create partnerships. In order to broaden the discussion on issues related to physical activity and public health, the International Congress on Physical Activity and Public Health (ICPAPH) was created. Epidemiological research in physical activity and health is growing in Brazil, as well as the participation of Brazilians on international events. The aim of this study was to describe the participation of Brazilians in every edition of the ICPAPH until 2014, when the event was held in Brazil. Through the analysis of programs of previous conferences, as well as the contact with the presidents of each edition and participants from Brazil, we noticed a participation from Brazilians since the very first edition, in 2006. Close to $2 \%$ of speakers and $5 \%$ of abstracts have been presented by Brazilian, with more participation in the event in Rio de Faneiro (14\% and 40\%, respectively), what was expected due to a large participation of locals in every edition of the event $(2006=68 \%$, $2008=14 \%, 2010=48 \%, 2012=60 \%$ and $2014=32 \%)$. Therefore, even though the participation of Brazilians has been growing, it is necessary to create strategies to increase Brazilian participation in international debates and financing for research in the field of physical activity and public bealth.
\end{abstract}

\section{KEYWORDS}

Research; Academies and institutes; Professional meetings and conferences.
Rev Bras Ativ Fís Saúde p. 65-72 $\mathrm{DOI}$

http://dx.doi.org/10.12820/rbafs.v.20n1p65

1 Programa de Pós-Graduação em Educação Física, Universidade Federal do Paraná

2 Programa de Pós-Graduação em

Endocrinologia, Universidade Federal do Rio Grande do Sul

3 Programa de Pós-Graduação em Educação Física, Universidade Federal de Pelotas

4 Programa de Pós-Graduação em Epidemiologia, Universidade Federal de Pelotas 


\section{INTRODUCCÃO}

A inatividade física é um problema de saúde pública. Estima-se que a cada ano 5,8 milhões de mortes no mundo são atribuídas à inatividade física ${ }^{1}$. Os estudos clássicos de Jeremy Morris ${ }^{2}$ e Ralph Paffenbarger ${ }^{3}$ foram os primeiros a associarem a prática de atividade física com redução nas taxas de mortalidade. Desde então, muitos foram os avanços na pesquisa em atividade física e saúde pública. Esta área de pesquisa vem passando por um crescimento acentuado em termos de produção científica, além de expandir seus enfoques preocupando-se com os níveis de atividade física da população ${ }^{4}$, seus determinantes ${ }^{5}$ e as modificações ao longo do tempo ${ }^{6}$, bem como intervenções populacionais para promoção de atividade física ${ }^{7}$ desenvolvendo conhecimento científico e tecnológico para alcançar esses objetivos.

Historicamente as pesquisas em atividade física e saúde pública se concentraram em países desenvolvidos ${ }^{8}$. Entretanto, observamos uma mudança neste cenário nos últimos anos. Neste contexto, acredita-se que América Latina tem muito a contribuir para o conhecimento sobre a área em questão ${ }^{9}$ destacando o Brasil, que é considerado um dos líderes mundiais na pesquisa em atividade física e saúde pública ${ }^{10}$.

$\mathrm{O}$ crescimento do número de pesquisas epidemiológicas em atividade física no Brasil foi expressivo a partir do ano $2000^{8}$. A participação em eventos científicos é um dos mecanismos de extrema importância no que diz respeito à atualização de pesquisadores. Neste espaço, a troca de experiências, a difusão de novos conceitos entre a comunidade científica, além da reflexão sobre o estado da arte de tópicos específicos podem contribuir significativamente para o crescimento de uma área de pesquisa.

Portanto, em 2006 foi criado o International Congress of Physical Activity and Public Health (ICPAPH), através dos esforços da International Society for Physical Activity and Health (ISPAH), com o objetivo de ampliar a discussão sobre questões relacionadas à prática de atividade física numa perspectiva de saúde pública. A primeira edição do congresso ocorreu em Atlanta-Estados Unidos (2006), sendo as edições subsequentes realizadas em Amsterdã-Holanda (2008), Toronto-Canadá (2010), Sidney-Austrália (2012) e Rio de Janeiro-Brasil (2014).

Mesmo o Brasil sendo um país com destaque internacional na produção científica na área de atividade física e saúde pública, pouco se sabe sobre a participação de brasileiros neste evento internacional. Assim, teve-se como objetivo descrever a inserção de pesquisadores brasileiros ao longo da trajetória do ICPAPH.

\section{MÉTODOS}

Trata-se de um estudo descritivo ${ }^{11}$, baseado no levantamento de informações referentes a todas as edições do ICPAPH. Para o levantamento dos dados foi realizado contato via e-mail com os presidentes das cinco edições do congresso e uma busca nas programações e anais dos eventos para acesso a informações adicionais.

Foram obtidos dados referentes ao tema principal e local de realização do evento, comissão organizadora (composto pela comissão geral do evento, 
comitê científico nacional e internacional), patrocinadores e apoiadores do evento, áreas temáticas das apresentações (modalidade oral e pôster), número de congressistas e palestrantes, número de conferências (abertura, encerramento e simpósios), mesas redondas, workshops e número de trabalhos aceitos (modalidade oral e pôster). Para os dados de participantes da comissão organizadora, congressistas, palestrantes e temas livres foram apresentados a participação total para outras nacionalidades e específica de brasileiros, de acordo com cada categoria.

A participação dos brasileiros, seja como palestrante ou congressista, nas edições do ICPAPH, foi analisada a partir dos cronogramas e anais dos eventos, e classificada de acordo com as seguintes áreas temáticas: prevalência de atividade física e tendências temporais; determinantes da prática de atividade física; consequências da prática de atividade física; intervenções para promoção da prática de atividade física; planos de ação local, regional e global; e outras áreas.

Simulações de custo para participação em congressos foram obtidas através de sites de busca para passagens aéreas e hospedagem, para datas semelhantes àquelas dos eventos, porém a busca foi realizada em abril de 2015 , não sendo possível realizar a busca para anos anteriores. Os custos foram calculados em dólares americanos (US\$).

Foi definido como participante brasileiro no evento, aquele que informou esta nacionalidade no momento da inscrição. Quando esta informação não estava disponível, o vínculo com uma instituição brasileira era considerado. Ainda, foram considerados congressistas locais nas outras edições aqueles com nacionalidade do país sede.

Todas as informações foram tabuladas em planilhas, sendo analisadas por meio de estatística descritiva com cálculos de frequências absolutas e relativas, através do software Microsoft Office Excel 2010.

\section{RESULTADOS}

Foram realizadas cinco edições do ICPAPH. Na tabela 1, observou-se maior número de palestrantes na primeira edição (178 participantes em Atlanta, 2006) em contraste com um menor número na edição do Rio de Janeiro (61). Notouse que a quantidade de atividades acadêmicas durante o evento científico foi similar em todas as edições, exceto na quarta edição, em Sidney (2012) devido à ocorrência de dois outros eventos científicos australianos paralelamente ao ICPAPH. Acredita-se que este fato também possa ter influenciado no número de participantes e apresentações de trabalhos na edição australiana do congresso. É marcante a proporção de congressistas locais, que ultrapassou $50 \%$ do total de participantes neste evento, similar ao primeiro, realizado em Atlanta.

A participação de brasileiros tem sido ativa desde a primeira edição do ICPAPH. Conforme mostra a tabela 2, em 2006 já havia ao menos um representante do Brasil na comissão organizadora do congresso. No mesmo ano, $1,2 \%(n=11)$ dos congressistas eram brasileiros, assim como $1,7 \%(n=3)$ do total de palestrantes. Todas as participações de palestrantes brasileiros estiveram relacionadas à intervenções para promoção da prática de atividade física. Em relação aos trabalhos apresentados, todos na forma de pôster, 46,7\% tratavam-se de estudos de prevalência de atividade física. No ano de 2012, 
em Sidney, com apenas dois palestrantes de origem brasileira, a participação foi de mais de 15\% em mesas redondas. Em 2014 o aumento foi ainda mais aparente (n=9;14,7\%), tendo em vista que 74\% da comissão organizadora era oriunda do país sede.

Ainda na tabela 2, há participação brasileira nos trabalhos apresentados nas modalidades oral e pôster, sem predominância de uma única área temática. Foram 15 os trabalhos apresentados em Atlanta, aumentando em mais de 100\% na segunda edição (34 trabalhos), repetindo a participação em Sidney. Apesar do aumento, estes trabalhos representaram menos de $10 \%$ do total de trabalhos em cada edição do evento. A maior proporção de apresentações esteve no Rio de Janeiro (7,8\% orais e 46,1\% pôsteres).

As palestras ministradas por brasileiros em um mesmo evento foram desenvolvidas dentro de temáticas diferentes. Já os trabalhos apresentados não foram predominantemente de um único eixo temático. Cabe destacar o fato de que estudos incluídos na área temática "planos de ação local, regional e

TABELA 1 - Total de palestrantes, congressistas e apresentações em todas as edições do International Congress on Physical Activity and Public Health.

\begin{tabular}{|c|c|c|c|c|c|c|c|}
\hline Edição & Ano & Cidade/País & Palestrantes & $\begin{array}{c}\text { Conferências/ } \\
\text { Mesas }\end{array}$ & Congressistas & $\begin{array}{c}\text { Congressistas } \\
\text { locais }^{c}\end{array}$ & Trabalhos \\
\hline 1 & 2006 & Atlanta, EUA & 178 & 73 & 884 & $603(68 \%)$ & 249 \\
\hline 2 & 2008 & $\begin{array}{l}\text { Amsterdã, } \\
\text { Holanda }\end{array}$ & 91 & 29 & 556 & $80(14 \%)$ & 503 \\
\hline 3 & 2010 & $\begin{array}{l}\text { Toronto, } \\
\text { Canadá }\end{array}$ & 104 & 33 & 1048 & $501(48 \%)$ & 573 \\
\hline 4 & 2012 & $\begin{array}{l}\text { Sidney, } \\
\text { Austráliab }^{\text {b }}\end{array}$ & 111 & 23 & 1114 & $667(60 \%)$ & 800 \\
\hline 5 & 2014 & $\begin{array}{l}\text { Rio de Janeiro, } \\
\text { Brasil }\end{array}$ & 61 & 20 & 503 & $177(35 \%)$ & 543 \\
\hline
\end{tabular}

atotal de apresentações orais e pôsteres

bevento concomitante a outros dois eventos científicos

'congressistas residentes no país de realização do evento

TABELA 2 - Número de participantes do International Congress on Physical Activity and Public Health de acordo com a edição e nacionalidade.

\begin{tabular}{|c|c|c|c|c|c|c|c|c|c|c|c|}
\hline \multirow{3}{*}{ Tipos de participação } & & \multicolumn{2}{|c|}{2006} & \multicolumn{2}{|c|}{2008} & \multicolumn{2}{|c|}{2010} & \multicolumn{2}{|c|}{2012} & \multicolumn{2}{|c|}{2014} \\
\hline & & \multicolumn{2}{|c|}{ Atlanta, EUA } & \multicolumn{2}{|c|}{$\begin{array}{l}\text { Amsterdã, } \\
\text { Holanda }\end{array}$} & \multicolumn{2}{|c|}{ Toronto, Canadá } & \multicolumn{2}{|c|}{ Sidney, Austrália } & \multicolumn{2}{|c|}{$\begin{array}{c}\text { Rio de Janeiro, } \\
\text { Brasil }\end{array}$} \\
\hline & & $\mathrm{n}$ & $\%$ & $\mathrm{n}$ & $\%$ & $\mathrm{n}$ & $\%$ & $\mathrm{n}$ & $\%$ & $\mathrm{n}$ & $\%$ \\
\hline \multirow[t]{2}{*}{ Comitê de Organização } & Brasil & 1 & 3,6 & $*$ & $*$ & 1 & 4,0 & 0 & 0 & 17 & 73,9 \\
\hline & Outros países & 27 & 96,4 & $*$ & $*$ & 24 & 96,0 & 36 & 100,0 & 6 & 26,1 \\
\hline \multirow[t]{2}{*}{ Congressistas } & Brasil & 11 & 1,2 & 13 & 2,2 & 29 & 2,8 & 31 & 2,8 & 226 & 32,3 \\
\hline & Outros países & 873 & 98,8 & 473 & 97,7 & 1019 & 97,2 & 1083 & 97,2 & 473 & 67,7 \\
\hline \multirow[t]{2}{*}{ Palestrantes } & Brasil & 3 & 1,7 & 3 & 3,3 & 4 & 3,8 & 2 & 1,8 & 9 & 14,7 \\
\hline & Outros países & 178 & 98,3 & 91 & 96,7 & 104 & 96,2 & 111 & 98,2 & 61 & 85,3 \\
\hline \multirow[t]{2}{*}{ Conferências/Mesas } & Brasil & 5 & 6,4 & 2 & 6,4 & 2 & 5,7 & 2 & 8,0 & 8 & 40,0 \\
\hline & Outros países & 73 & 93,6 & 29 & 93,6 & 33 & 95,3 & 23 & 92,0 & 20 & 60,0 \\
\hline \multirow[t]{2}{*}{ Trabalhos (Oral) } & Brasil & 0 & 0,0 & 7 & 3,5 & 0 & 0 & 14 & 2,8 & 15 & 7,8 \\
\hline & Outros países & 0 & 0,0 & 195 & 96,5 & 48 & 100,0 & 486 & 97,2 & 177 & 92,2 \\
\hline \multirow[t]{2}{*}{ Trabalhos (Pôster) } & Brasil & 15 & 6,0 & 27 & 8,9 & 20 & 3,8 & 20 & 6,7 & 162 & 46,1 \\
\hline & Outros países & 234 & 94,0 & 274 & 91,1 & 505 & 96,2 & 280 & 93,3 & 189 & 53,9 \\
\hline
\end{tabular}

dados indisponíveis 
global” foram introduzidos somente na edição do Rio de Janeiro e, portanto, não havia sido contemplada até então por parte dos pesquisadores brasileiros, e representaram 3,2\% dos trabalhos apresentados no evento (Tabela 2).

Ao longo dos anos, notou-se declínio no número de patrocinadores (27 em 2006; cinco em 2014), assim como no número de áreas temáticas para apresentação de trabalhos (23 em 2008; seis em 2014).

A tabela 3 indica estimativas de custo para que um brasileiro pudesse participar dos eventos em cada ano e, como esperado, o evento europeu demandou maiores gastos, bem como o primeiro evento em Atlanta e aquele em Sidney. Participar em Amsterdã foi considerado o menos acessível (US\$3.636,00/ pessoa) e Rio de Janeiro o mais acessível (US\$1.540,00/pessoa). O próximo congresso, a ser realizado na Tailândia, não estará entre os mais caros.

TABELA 3 - Estimativa de custo para participação de um brasileiro no International Congress on Physical Activity and Public Health.

\begin{tabular}{lcccc}
\hline Local do evento & $\begin{array}{c}\text { Passagem aérea } \\
\text { (US\$) }\end{array}$ & $\begin{array}{c}\text { Hospedagem } \\
\text { (US\$) }\end{array}$ & $\begin{array}{c}\text { Inscrição } \\
\text { (US\$) }\end{array}$ & $\begin{array}{c}\text { Total } \\
\text { (US\$) }\end{array}$ \\
\hline Atlanta, EUA & 1945,00 & 420,00 & 1000,00 & 3365,00 \\
\hline Amsterdã, Holanda & 1722,00 & 914,00 & 1000,00 & 3636,00 \\
\hline Toronto, Canadá & 566,00 & 521,00 & 1000,00 & 2087,00 \\
\hline Sidney, Austrália & 1599,00 & 413,00 & 1000,00 & 3012,00 \\
\hline Rio de Janeiro, Brasil & 152,00 & 388,00 & 1000,00 & 1540,00 \\
\hline Bangkok, Tailândia & 922,00 & 341,00 & 1000,00 & 2263,00 \\
\hline
\end{tabular}

a valor de passagem área com saída de São Paulo-SP, conforme busca realizada em (www.decolar.com), em 14/04/2015

${ }^{\mathrm{b}}$ preço médio para cinco diárias em hotéis com três estrelas. Fonte: www.booking.com

c valor médio de inscrição estimado com base nos eventos de 2012 e 2014.

Ainda, o número de membros da Sociedade Internacional de Atividade Física e Saúde (ISPAH) em 2014 era de 181, sendo apenas quatro brasileiros (2,2\%). A Sociedade Brasileira de Atividade Física e Saúde (SBAFS) contava com 131 membros ativos no mesmo período.

\section{DISCUSSÃO}

Este estudo teve como objetivo descrever a participação de brasileiros nas cinco edições do ICPAPH. Durante as últimas edições do evento foi possível observar uma mudança estrutural do mesmo, com a redução no número de palestras e workshops, os quais cederam espaço para sessões científicas, formatação que foi mantida no evento no Rio de Janeiro. Em relação à participação do Brasil, observou-se um crescimento no número de congressistas ao longo das edições, enquanto a participação em sessões científicas manteve-se sempre abaixo de $10 \%$ do total de estudos apresentados. Na edição do Rio de Janeiro marcaram presença mais de 200 congressistas brasileiros, com participação em $46,1 \%$ das sessões de pôster e 7,8\% das apresentações orais. Apesar de ambos serem apresentados na língua inglesa, as sessões orais demandam uma fala mais extensa e formal. Portanto, acredita-se que o idioma pode ter sido um fator de influência na escolha do tipo de trabalho a ser apresentado pelos brasileiros.

A realização de congressos e eventos científicos em geral é de extrema importância para a comunidade cientifica ${ }^{12}$. A oportunidade de reunir em um 
único local pesquisadores de excelência com congressistas do mundo todo favorece o crescimento em qualquer área cientifica. Em se tratando de atividade física e saúde pública a realização de eventos deste nível permite com que avanços nesta área aconteçam. É a partir dos congressos científicos que decisões e metas de pesquisas são tomadas. Para o Brasil, ter a oportunidade de sediar um evento deste porte oportunizou aos pesquisadores, estudantes de graduação e pós-graduação residentes no Brasil a convivência, troca de informações, experiências e parcerias com os maiores nomes do mundo na área de atividade física e saúde pública ${ }^{10}$. Além disso, trazer o ICPAPH ao Brasil voltou os "olhos" da comunidade científica mundial sobre o problema da inatividade física e sedentarismo, que afetam principalmente países de renda média ou baixa, conforme comentários de pesquisadores envolvidos neste evento desde sua primeira edição (Figura 1).

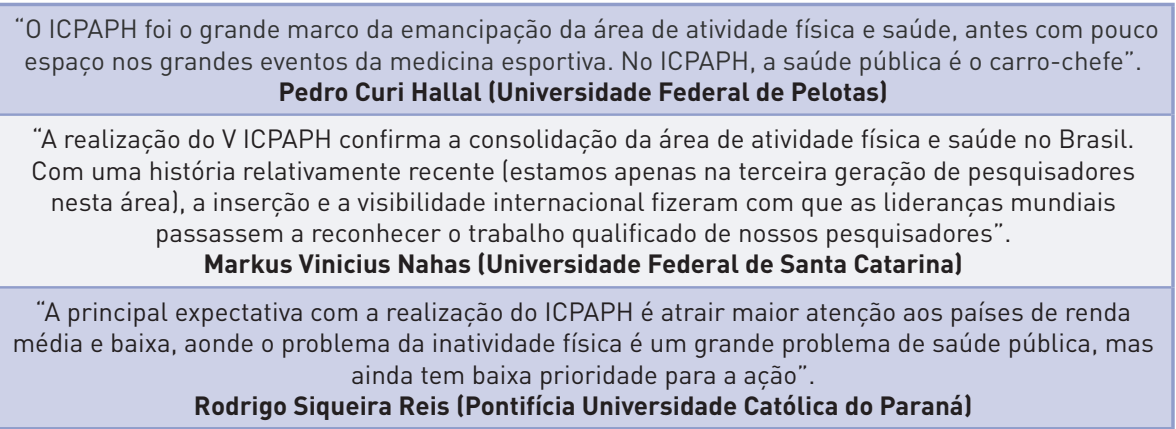

FIGURA 1 - Relatos de brasileiros participantes de diversas edições do International Congress on Physical Activity and Public Health.

A participação brasileira no ICPAPH vem aumentando com o passar das edições em paralelo com o crescimento da área de pesquisa em atividade física e saúde pública no país. Desde a primeira edição do evento, em Atlanta, até a última no Rio de Janeiro, observou-se um aumento de cerca de três vezes no número de brasileiros inscritos. Da mesma forma, a representação de brasileiros em espaços de apresentação de trabalhos aumentou consideravelmente com o passar dos anos. Por exemplo, enquanto na primeira edição foram apresentados 15 trabalhos, todos na modalidade pôster, na última edição esse número passou para 177, sendo 15 deles apresentados de forma oral, aumento já esperado pela realização do evento no Brasil.

Entre todas as edições do ICPAPH, 10 palestrantes eram de nacionalidade brasileira e somente dois brasileiros participaram da organização dos congressos anteriores, realizados nos países desenvolvidos. A representação brasileira nos congressos pode ser interpretada numa perspectiva otimista ou pessimista. Se por um lado todas as edições do evento contaram com baixa participação de brasileiros, por outro lado, observamos um aumento expressivo no número de brasileiros participando de diferentes espaços de debate com o passar dos anos. Acredita-se que as principais barreiras para a pequena participação no evento podem ser o alto custo financeiro e o idioma oficial adotado nos congressos.

Desde a primeira edição e independente do local de realização, observamos que o investimento necessário para participação é de aproximadamente US\$ 3.000,00 (Tabela 3). Embora o Conselho Nacional de Desenvolvimento 
Científico e Tecnológico $(\mathrm{CNPq})$ forneça auxílio em diárias para apoiar a participação de pesquisadores brasileiros em eventos científicos com abrangência nacional e internacional, é possível que este valor não seja compatível com o total de investimento necessário. Mesmo na edição a ser realizada em 2016, na Tailândia, os custos não são superiores a outras edições realizadas em localidades de mais fácil acesso. Portanto, poderia se esperar uma participação ainda muito imponente da comunidade brasileira no próximo evento.

Para tanto, é necessário fomentar de forma interligada a captação de financiamento e o auxílio para a participação em eventos dentro da área de atividade física e saúde. A expansão da captação de recursos para além dos centros de excelência na área, bem como a diversificação das fontes de financiamento exploradas são metas que precisam ser perseguidas nos próximos anos para o fortalecimento de pesquisa na área e fomento de relatos cada vez mais qualificados, questões que devem impactar de forma direta na participação brasileira no principal congresso da área.

O idioma também está entre as barreiras para participação dos brasileiros no evento. A adoção de tradução para língua local, principalmente quando o evento for realizado em países emergentes, poderia ser uma estratégia provisória para aumentar a participação de congressistas nacionais. $\mathrm{O}$ incentivo ao aprendizado da língua inglesa é tratado como uma questão estratégica pelo Ministério da Educação (MEC) para o desenvolvimento da pós-graduação no Brasil, no entanto, outros esforços são necessários. Entre eles pode-se apontar a criação de estratégias individuais pelos programas de pós-graduação para o fomento do aprendizado da língua inglesa, aumentando-se o nível de exigência para entrada nos mesmos.

Ainda, a Sociedade Internacional de Atividade Física e Saúde (ISPAH) e a Sociedade Brasileira de Atividade Física e Saúde (SBAFS) podem ser mecanismos de apoio à participação de brasileiros na comunidade científica mundial uma vez que estão diretamente vinculadas a este e outros eventos científicos da área. A baixa participação dos brasileiros internacionalmente $(n=4)$ poderia ser um indicador da pouca inserção do país no ICPAPH até o momento, uma vez que a SBAFS contava com 131 membros ativos no mesmo período.

A participação dos brasileiros na comunidade científica da atividade física e saúde tem crescido ao longo dos anos, principalmente no ICPAPH, mas ainda é pouco representativa da inserção mundial nesta área de pesquisa. Sendo assim, percebe-se a necessidade de fortalecer e ampliar a participação de pesquisadores e principalmente alunos de pós-graduação no ICPAPH para difundir ainda mais o trabalho realizado. Nesse sentido, temas como o apoio para participação em eventos, financiamento de pesquisa e qualificação profissional, precisam entrar de forma prioritária na pauta da área.

Ainda, esta maior representatividade brasileira somente poderá ser considerada consolidada uma vez que as tendências verificadas neste estudo continuem a ser positivas. Portanto, devemos aguardar o evento a ser realizado na Tailândia para confirmar se seguimos adiante e para cima ou se fomos vítimas da casualidade.

\section{REFERÊNCIAS}

1. Lee I, Shiroma E, Labelo F, Puska P, Blair S, Katzmarzyk P. Effect of physical inactivity on major non-communicable diseases worldwide: an analysis of burden of disease and life expectancy. Lancet. 2012:9-19. 
2. Morris JN, Heady JA, Raffle PA, Roberts CG, Parks JW. Coronary heart-disease and physical activity of work. Lancet. Nov 28 1953;265(6796):1111-1120; concl.

3. Paffenbarger RS, Jr., Wing AL, Hyde RT. Physical activity as an index of heart attack risk in college alumni. Am J Epidemiol. Sep 1978;108(3):161-175.

4. Hallal PC, Andersen LB, Bull FC, Guthold R, Haskell W, Ekelund U. Global physical activity levels: surveillance progress, pitfalls, and prospects. Lancet. Jul 21 2012;380(9838):247-257.

5. Bauman AE, Reis RS, Sallis JF, Wells JC, Loos RJ, Martin BW. Correlates of physical activity: why are some people physically active and others not? Lancet. Jul 21 2012;380(9838):258-271.

6. Morseth B, Jorgensen L, Emaus N, Jacobsen BK, Wilsgaard T. Tracking of leisure time physical activity during $28 \mathrm{yr}$ in adults: the Tromso study. Med Sci Sports Exerc. Jul 2011;43(7):1229-1234.

7. Heath GW, Parra DC, Sarmiento OL, et al. Evidence-based intervention in physical activity: lessons from around the world. Lancet. Jul 21 2012;380(9838):272-281.

8. Hallal PC, Dumith SC, Bastos JP, Reichert FF, Siqueira FV, Azevedo MR. Evolution of the epidemiological research on physical activity in Brazil: a systematic review. Rev Saúde Pública. Jun 2007;41(3):453-460.

9. Hallal P, Parra D, Azevedo M, Pratt M, Brownson R. Research on Physical Activity and Health: Where is Latin America? J Phys Act Health. 2010;7(Suppl 2):S129-S130.

10. Reis RS. International Congress on Physical Activity and Public Health: Atlanta, Amsterdam, Toronto, Sydney, next stop, Rio de Janeiro, Brazil. Rev Bras Ativ Física Saúde. 10 2012;17(5).

11. Jekel JF, Elmore JG. Epidemiologia, Bioestatística e Medicina Preventiva. Porto Alegre; 1999.

12. Katzmarzyk P. The Third International Congress on Physical Activity and Public Health: Toronto, May 5-8, 2010. J Phys Act Health. 2010;7(Suppl 3):S281-S282.

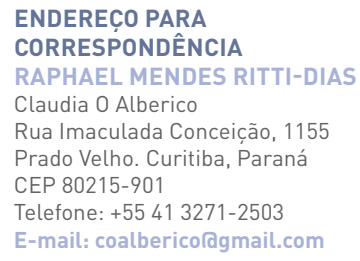

